\title{
On fractional Euler-Lagrange and Hamilton equations and the fractional generalization of total time derivative
}

\author{
Dumitru Baleanu ${ }^{1}$ \\ Department of Mathematics and Computer Sciences, Faculty of Arts and \\ Sciences, Çankaya University- 06530, Ankara, Turkey \\ Sami I. Muslih ${ }^{2}$ \\ Department of Physics, Al-Azhar University, Gaza, Palestine \\ and \\ International Center for Theoretical Physics, Trieste, Italy \\ Eqab M. Rabei \\ Department of Science-Jerash Private University, Jerash-Jordan \\ and \\ Department of Physics-Mutah University, Karak-Jordan
}

\begin{abstract}
Fractional mechanics describes both conservative and non-conservative systems. The fractional variational principles gained importance in studying the fractional mechanics and several versions are proposed. In classical mechanics the equivalent Lagrangians play an important role because they admit the same Euler-Lagrange equations. By adding a total time derivative of a suitable function to a given classical Lagrangian or by multiplying with a constant the Lagrangian we obtain the same equations of motion. In this study, the fractional discrete Lagrangians which differs by a fractional derivative are analyzed within Riemann-Liouville fractional derivatives. As a consequence of applying this procedure the classical results are reobtained as a special case.

The fractional generalization of Faà di Bruno formula is used in order to obtain the concrete expression of the fractional Lagrangians which differs from a given fractional Lagrangian by adding a fractional derivative. The fractional Euler-Lagrange and Hamilton equations corresponding to the obtained fractional Lagrangians are investigated and two examples are analyzed in details.
\end{abstract}

Keywords: fractional Lagrangians, fractional calculus, fractional Riemann-Liouville derivative, fractional Euler-Lagrange equations, Faà di Bruno formula.

\section{Introduction}

Fractional calculus is a generalization of ordinary differentiation and integration to arbitrary order.The fractional derivatives are the infinitesimal generators of

\footnotetext{
${ }^{1}$ On leave of absence from Institute of Space Sciences, P.O.BOX, MG-23, R 76900, MagureleBucharest, Romania,E-mails: dumitru@cankaya.edu.tr, baleanu@venus.nipne.ro

${ }^{2}$ E-mail: smuslih@ictp.trieste.it
} 
a class of translation invariant convolution semigroups which appear universally as attractors. Various applications of fractional calculus are based on replacing the time derivative in an evolution equation with a derivative of fractional order. The results of several recent researchers confirm that fractional derivatives seem to arise for important mathematical reasons. During the last decades the fractional calculus [1-3] started to be used in various fields, e.g. engineering, physics, biology and many important results were reported [4-19]. A special attention has been devoted during the last years to the fractional variational principles [20-29] and their applications [30-35]. For the constrained systems [36] the fractional Lagrangian and Hamiltonian formalism are still at the beginning of their development. The formulation of the fractional variational principles still needs to be more elaborated in the future and it will have an important impact on the elaboration of a consistent fractional quantization method for both discrete and continuous systems. The fractional variational principles is deeply related to the fractional quantization procedure. For a given Lagrangian there are several proposed methods to obtain the fractional Euler-Lagrange equations and the corresponding Hamiltonians. However this issue is not yet complectly clarified and it requires more further detailed analysis. One of the main obstacles is related to the fractional Leibniz rule, the fractional chain rule [37] as well as the fractional Taylor series $[38,39]$. The second problem is related to the non-locality of the fractional Lagrangian.Recently, the Hamiltonian formalism for nonlocal Lagrangians was investigated in [40] and a relation of non-local theories and Ostrogradski's formalism [41, 42] was reported [43],[44].

As it is well known the classical equivalent Lagrangians represent a powerful tool for studying the Hamilton-Jacobi equation in Carathéodory's formulation [45] as well as in generalized mechanics [46, 47].

For these reasons, in this study we generalized the notion of equivalent Lagrangian for the fractional case. The fractional Euler-Lagrange equations of fractional equivalent Lagrangians are calculated and the fractional Hamiltonians are constructed.

The plan of this paper is as follows:

In Section 2, some basic formulas of the fractional calculus are briefly reviewed. Section 3 briefly review the fractional Hamiltonian approach of discrete systems. In Section 4 the fractional Lagrangians are investigated and two examples are investigated. Section 5 is dedicated to our conclusions.

\section{Brief overview of fractional calculus}

In this section, we formulate the problem in terms of the left and the right Riemann-Liouville (RL) fractional derivatives, which are defined as follows: the left Riemann-Liouville fractional derivative 


$$
{ }_{a} \mathbf{D}_{t}^{\alpha} f(t)=\frac{1}{\Gamma(n-\alpha)}\left(\frac{d}{d t}\right)^{n} \int_{a}^{t}(-\tau+t)^{n-\alpha-1} f(\tau) d \tau,
$$

and the right Riemann-Liouville fractional derivative

$$
{ }_{t} \mathbf{D}_{b}^{\alpha} f(t)=\frac{1}{\Gamma(n-\alpha)}\left(-\frac{d}{d t}\right)^{n} \int_{t}^{b}(\tau-t)^{n-\alpha-1} f(\tau) d \tau,
$$

where the order $\alpha$ fulfills $n-1 \leq \alpha<n$ and $\Gamma$ represents the Euler's Gamma function. It is observed that if $\alpha$ becomes an integer, we recovered the usual definitions, namely,

$$
{ }_{a} \mathbf{D}_{t}^{\alpha} f(t)=\left(\frac{d}{d t}\right)^{\alpha} f(t), \quad{ }_{t} \mathbf{D}_{b}^{\alpha} f(t)=\left(-\frac{d}{d t}\right)^{\alpha} f(t), \alpha=1,2, \ldots
$$

Fractional RL derivatives have many interesting properties. By direct calculation we observe that the RL derivative of a constant is not zero, namely

$$
{ }_{a} \mathbf{D}_{t}^{\alpha} C=C \frac{(t-a)^{-\alpha}}{\Gamma(1-\alpha)} .
$$

$\mathrm{RL}$ derivative of a power of $\mathrm{t}$ has the following form

$$
{ }_{a} \mathbf{D}_{t}^{\alpha} t^{\beta}=\frac{\Gamma(\alpha+1) t^{\beta-\alpha}}{\Gamma(\beta-\alpha+1)}
$$

for $\alpha>-1, \beta \geq 0$. Composite of fractional derivatives is given by the following formula

$$
{ }_{a} \mathbf{D}_{t a}^{\alpha} \mathbf{D}_{t}^{\sigma} f(t)={ }_{a} \mathbf{D}_{t}^{\alpha+\sigma} f(t)-\left.\sum_{j=1}^{k}{ }_{a} \mathbf{D}_{t}^{\sigma-j} f(t)\right|_{t=a} \frac{(t-a)^{-\alpha-j}}{\Gamma(1-\alpha-j)},
$$

where $0 \leq k-1 \leq q \leq k, p \geq 0$ and $\mathrm{k}$ is a whole number. As it can be seen from (6) the composition is not commutative. Finally, the fractional product rule is given below

$$
{ }_{a} \mathbf{D}_{t}^{\alpha}(f g)=\sum_{j=0}^{\infty}\left(\begin{array}{c}
\alpha \\
j
\end{array}\right)\left({ }_{a} \mathbf{D}_{t}^{\alpha-j} f\right)\left(\frac{d^{i} g}{d t^{j}}\right) .
$$

By inspection we observe that the fractional product contains infinitely many terms and this product is taking into account the memory.

\subsection{Fractional derivative of a composite function}

In order to find the fractional generalization of a composition function we have obtain first of all the most general classical counterpart. 
Let us take an analytic function $\phi(t)$ and $f(t)=H(t-a)$, where $H(t)$ is the Heaviside function. Using the Leibniz rule and the formula for the fractional differentiation of the Heaviside function we obtain

$$
{ }_{a} \mathbf{D}_{t}^{p} \phi(t)=\sum_{k=0}^{\infty}\left(\begin{array}{c}
p \\
k
\end{array}\right) \phi^{(k)}(t){ }_{a} \mathbf{D}_{t}^{p-k} H(t-a)
$$

or

$$
{ }_{a} \mathbf{D}_{t}^{p} \phi(t)=\frac{(t-a)^{-p}}{\Gamma(1-p)} \phi(t)+\sum_{k=1}^{\infty}\left(\begin{array}{c}
p \\
k
\end{array}\right) \frac{(t-a)^{k-p}}{\Gamma(k-p+1)} \phi^{(k)}(t)
$$

under the assumption $t>a$. Let us suppose that $\phi(t)$ is a composite function

$$
\phi(t)=F(h(t)) .
$$

The k-th order derivative of $\phi(t)$ is evaluated with the help of the Fa⿳亠 di Bruno formula [37]

$$
\frac{d^{k}}{d t^{k}} F(h(t))=k ! \sum_{m=1}^{k} F^{(m)}(h(t)) \sum \prod_{r=1}^{k} \frac{1}{a_{r} !}\left(\frac{h^{(r)}(t)}{r !}\right)^{a_{r}},
$$

where the sum $\sum$ extends over all combinations of non-negative integer values of $a_{1}, \cdots, a_{k}$ such that

$$
\sum_{r=1}^{k} r a_{r}=k
$$

and

$$
\sum_{r=1}^{k} a_{r}=m
$$

Therefore, the fractional derivative of a composition function is given by

$$
\begin{aligned}
& { }_{a} \mathbf{D}_{t}^{p} F(h(t))=\frac{(t-a)^{-p}}{\Gamma(1-p)} F(h(t))+ \\
& \sum_{k=1}^{\infty}\left(\begin{array}{c}
p \\
k
\end{array}\right) \quad k ! \frac{(t-a)^{k-p}}{\Gamma(k-p+1)} \sum_{m=1}^{k} F^{(m)}(h(t)) \sum \prod_{r=1}^{k} \frac{1}{a_{r} !}\left(\frac{h^{(r)}(t)}{r !}\right)^{a_{r}}
\end{aligned}
$$

where the sum $\sum$ and coefficients $a_{r}$ have the meaning explained above [2].

\section{Fractional Lagrangian and Hamiltonian analysis of discrete systems}

For a given fractional Lagrangian given by

$$
L_{f}\left(q^{\rho}(t),{ }_{a} \mathbf{D}_{t}^{\alpha} q^{\rho}(t),{ }_{t} \mathbf{D}_{b}^{\alpha} q^{\rho}(t)\right), \rho=1, \cdots, N
$$


the Euler-Lagrange equations are given as follows (see for example Refs. $[22],[28]$ and the references therein)

$$
\frac{\partial L_{f}}{\partial q^{\rho}(t)}+{ }_{t} \mathbf{D}_{b}^{\alpha} \frac{\partial L_{f}}{\partial_{a} \mathbf{D}_{t}^{\alpha} q^{\rho}(t)}+{ }_{a} \mathbf{D}_{t}^{\alpha} \frac{\partial L_{f}}{\partial_{t} \mathbf{D}_{b}^{\alpha} q^{\rho}(t)}=0,0<\alpha<1
$$

For simplicity,in the following we consider the following form of the fractional Euler-Lagrange equations

$$
\frac{\partial L_{f}^{\prime}}{\partial q^{\rho}(t)}+{ }_{t} \mathbf{D}_{b}^{\alpha} \frac{\partial L_{f}^{\prime}}{\partial_{a} \mathbf{D}_{t}^{\alpha} q^{\rho}(t)}=0,0<\alpha<1, \rho=1, \cdots, N
$$

In the following by using (17) we define the generalized momenta as (see Ref.[27] for more details)

$$
p_{\alpha_{\rho}}=\frac{\partial L_{f}^{\prime}}{\partial_{a} \mathbf{D}_{t}^{\alpha} q^{\rho}(t)}, \rho=1, \cdots, N
$$

As a consequence of (17) and (18) a Hamiltonian function is defined as

$$
H=p_{\alpha_{\rho} a} \mathbf{D}_{t}^{\alpha} q^{\rho}(t)-L_{f}^{\prime} .
$$

The canonical equations corresponding to (19) are given below

$$
\frac{\partial H}{\partial t}=-\frac{\partial L_{f}^{\prime}}{\partial t}, \frac{\partial H}{\partial p_{\alpha_{\rho}}}={ }_{a} \mathbf{D}_{t}^{\alpha} q^{\rho}, \frac{\partial H}{\partial q^{\rho}}={ }_{t} \mathbf{D}_{b}^{\alpha} p_{\alpha_{\rho}}, 0<\alpha<1, \rho=1, \cdots, N .
$$

We mention that other interesting formulations of fractional Lagrangian and Hamiltonian dynamics can be found in [20, 21] and [23].

\section{Fractional derivatives and fractional Lagrangian mechanics}

Let $L\left(q^{\rho}(t), \dot{q}^{\rho}(t)\right)$ be a classical Lagrangian function with $\rho=1, \cdots, N$. Let $L^{\prime}\left(q^{\rho}, \dot{q}^{\rho}(t)\right)=L\left(q^{\rho}(t), \dot{q}^{\rho}(t)\right)+\frac{d F\left(q^{m}(t)\right)}{d t}$, where $F\left(q^{m}(t)\right)$ is a differentiable function and $q^{m}(t)$ is one of the coordinates. It is very well known that two equivalent classical Lagrangians admit the same Euler-Lagrange equations. In the following we are considering the fractional generalization of this classical results, namely we replace the normal derivatives by the fractional ones. 


\subsection{A particular case}

One of the possible generalizations of the classical Lagrangian $L\left(q^{\rho}(t), \dot{q}^{\rho}(t)\right)$ is given by

$$
L_{f}\left(q^{\rho}(t),{ }_{a} \mathbf{D}_{t}^{\alpha} q^{\rho}(t)\right) .
$$

If we add to (21) a term of the form ${ }_{t} \mathbf{D}_{b}^{\alpha} q(t)$ we obtain

$$
L_{f}^{\prime}=L_{f}\left(q^{\rho}(t),{ }_{a} \mathbf{D}_{t}^{\alpha} q^{\rho}(t)\right)+C_{a} \mathbf{D}_{t}^{\alpha} q^{m}(t),
$$

where $m \in\{1, \cdots, N\}$ is a chosen coordinate and $\mathrm{C}$ is a non-zero real constant. The fractional Euler-Lagrange equations of (22) are given by

$$
\begin{gathered}
\frac{\partial L_{f}}{\partial q^{\sigma}(t)}+{ }_{t} \mathbf{D}_{b}^{\alpha} \frac{\partial L_{f}}{\partial_{a} \mathbf{D}_{t}^{\alpha} q^{\sigma}(t)}=0, \\
\frac{\partial L_{f}}{\partial q^{m}(t)}+{ }_{t} \mathbf{D}_{b}^{\alpha} \frac{\partial L_{f}}{\partial_{a} \mathbf{D}_{t}^{\alpha} q^{m}(t)}+C \frac{(b-t)^{-\alpha}}{\Gamma(1-\alpha)}=0, \\
\frac{\partial L_{f}}{\partial q^{\delta}(t)}+{ }_{t} \mathbf{D}_{b}^{\alpha} \frac{\partial L_{f}}{\partial_{a} \mathbf{D}_{t}^{\alpha} q^{\delta}(t)}=0,
\end{gathered}
$$

where $\sigma=1, \cdots, m-1$ and $\delta=m+1, \cdots, N$.

The last term of (24) arises after taking into account (16) and (4) with $C=1$.

\subsubsection{An example}

Let us consider the fractional generalization of a free Lagrangian of one degree of freedom $L=\frac{\dot{x}^{2}}{2}$ at which we add a term of the form $\dot{x}$. At the classical level the Lagrangians $L=\frac{\dot{x}^{2}}{2}$ and $L^{\prime}=\frac{\dot{x}^{2}}{2}+\dot{x}$ are equivalent.

One of the possible generalizations for the fractional case is given below

$$
L_{f}=\frac{\left({ }_{a} \mathbf{D}_{t}^{\alpha} q(t)\right)^{2}}{2}+C_{a} \mathbf{D}_{t}^{\alpha} q(t),
$$

where $\mathrm{C}$ is a non-zero real constant.

By using (26) the fractional equation of motion is given bellow

$$
{ }_{t} \mathbf{D}_{b}^{\alpha}\left({ }_{a} \mathbf{D}_{t}^{\alpha} q(t)\right)+C \frac{(b-t)^{-\alpha}}{\Gamma(1-\alpha)}=0 .
$$

From (26) the expression of the fractional canonical momentum is

$$
p_{\alpha}={ }_{a} \mathbf{D}_{t}^{\alpha} q(t)+C .
$$

The fractional canonical Hamiltonian is given by

$$
H_{f}=p_{\alpha a} \mathbf{D}_{t}^{\alpha} q(t)-L_{f}=\frac{\left(p_{\alpha}-C\right)^{2}}{2} .
$$


Applying the method developed in [27] we obtain

$$
\frac{\partial H}{\partial p_{\alpha}}={ }_{a} \mathbf{D}_{t}^{\alpha} q(t), \frac{\partial H}{\partial q}={ }_{t} \mathbf{D}_{b}^{\alpha} p_{\alpha}
$$

or

$$
{ }_{a} \mathbf{D}_{t}^{\alpha} q(t)=p_{\alpha}-C,{ }_{t} \mathbf{D}_{b}^{\alpha} p_{\alpha}=0 .
$$

Therefore, from (31) and (27) and taking into account (28) we conclude that fractional Euler-Lagrange and fractional Hamilton equations give the same evolution equation for the coordinate $\mathrm{q}(\mathrm{t})$. Besides, when the constant $\mathrm{C}$ becomes zero and $\alpha \rightarrow 1$ the classical results are reobtained.

\subsection{The general case}

The next step is to add to the fractional Lagrangian $L_{f}\left(q^{\rho}(t),{ }_{a} \mathbf{D}_{t}^{\alpha} q^{\rho}(t)\right), \rho=$ $1, \cdots, N$ a most general term which under some limits becomes the classical total derivative. We analyze the fractional equivalent Lagrangians as it is given below

$$
L_{f}^{\prime}=L_{f}\left(q^{\rho}(t),{ }_{a} \mathbf{D}_{t}^{\alpha} q^{\rho}(t)\right)+{ }_{a} \mathbf{D}_{t}^{\alpha} F\left(q^{m}(t)\right), \rho=1, \cdots, n .
$$

By using the fractional generalization of Faà di Bruno formula given in (14) we obtain the explicit form of (32) as follows

$$
\begin{gathered}
L_{f}^{\prime}=\quad L_{f}\left(q^{\rho}(t),{ }_{a} \mathbf{D}_{t}^{\alpha} q^{\rho}(t)\right)+\frac{(t-a)^{-\alpha}}{\Gamma(1-\alpha)} F\left(q^{m}(t)\right)+ \\
\sum_{k=1}^{\infty}\left(\begin{array}{c}
\alpha \\
k
\end{array}\right) \quad k ! \frac{(t-a)^{k-\alpha}}{\Gamma(k-\alpha+1)} \sum_{s=1}^{k} F^{(s)}\left(q^{m}(t)\right) \sum \prod_{r=1}^{k} \frac{1}{a_{r} !}\left(\frac{\left(q^{m}\right)^{(r)}(t)}{r !}\right)^{a_{r}} .
\end{gathered}
$$

The presence of the infinite higher order derivatives in (33) rises an interesting question regarding the form of the corresponding Euler-Lagrange equations as well as the form of the corresponding Hamiltonian construction. The theory described by (33) is non-local and involve infinity derivative of $q(t)$. The key point is to use the formula (9) which allowed to represent the both parts of the Lagrangian in a common way.

The next step is to write the Euler-Lagrange equations and the corresponding Hamiltonian.

In this line of taught we consider that the dynamical variable $q(t)$ is a $1+1$ dimensional field $Q(x, t)$ subjected to the following chirality condition [43]

$$
\frac{d Q(x, t)}{d t}=\partial_{x} Q(x, t)
$$

A specific feature of the Hamiltonian formalism for non-local theories is that is contains the Euler-Lagrange equations as Hamiltonian constraints. 
By using (34) we have

$$
\left(\frac{d}{d t}\right)^{n} Q(x, t)=\left(\partial_{x}\right)^{n} Q(x, t), n \in N_{0},
$$

and having in mind that $Q(x, t)=q(x+t)$ assures the one-to-one correspondence between $q(t)$ and $Q(x, t)$ [43]. Ostrogradski's coordinates are defined as follows:

$$
Q^{(n)}(t)=\left.\left(\partial_{x}\right)^{n} Q(x, t)\right|_{x=x_{0}},
$$

where the discontinuity curve $x_{0}(t)=x_{0}$ is a constant [43].

By using the inverse relation provided by the Taylor expansion around $x=x_{0}$ we obtain

$$
Q(x, t)=\sum_{n=0}^{\infty} \frac{\left(x-x_{0}\right)^{n}}{n !} Q^{(n)}(t) .
$$

We notice that we reobtain the chirality condition as

$$
\dot{Q}^{(n)}(t)=Q^{(n+1)}(t) .
$$

A boundary Poisson bracket was introduced in [43] and it has the expression as follows

$$
\{F(t), G(t)\}=\sum_{k, l=0}^{\infty} c_{k, l} \int_{-\infty}^{\infty} d x\left(\partial_{x}\right)^{k+l}\left[\frac{\delta F(t)}{\delta Q^{(k)}}(x, t) \frac{\delta G(t)}{\delta P^{(l)}}(x, t)\right]-(F \leftrightarrow G),
$$

where the coefficients $c_{k, l}$ are constants and normalized in such a manner to satisfy the Jacobi identity. Fixing the time the classical canonical relation is naturally obtained from (39) as

$$
\left\{Q(x, t), P\left(x^{\prime}, t\right)\right\}=\delta_{R}\left(x-x^{\prime}\right) .
$$

By using (39) and (40) and defining Ostrogradski's momenta $P_{(n)}(t)$ as

$$
P_{(n)}(t)=\int_{-\infty}^{\infty} d x \frac{\left(x-x_{0}\right)^{n}}{n !} P(x, t) .
$$

From (41) the form of $P(x, t)$ is given by

$$
P(x, t)=\sum_{n=0}^{\infty} P_{(n)}(t)\left(-\partial_{x}\right)^{n} \delta_{R}\left(x-x_{0}\right)
$$

By using (42) the expression for $P_{(n)}(t)$ is as follows

$$
P_{(n)}(t)=\sum_{m=n}^{\infty}\left(-\partial_{t}\right)^{m-n} \frac{\partial L_{f}^{\prime}[Q](t)}{\partial Q^{(m+1)}(t)} .
$$


The above definitions leads us to the following Hamilton equations

$$
\begin{gathered}
\dot{P}_{(n)}(t)+P_{(n-1)}(t)=\frac{\partial L_{f}^{\prime}[Q](t)}{\partial Q^{(n)}(t)}, n \in N, \\
\dot{P}_{(0)}(t)=\frac{\partial L_{f}^{\prime}[Q](t)}{\partial Q^{(0)}(t)} .
\end{gathered}
$$

Taking into account the definition of $P_{(0)}(t)$ we observe that (45) is the EulerLagrange equation corresponding to the lagrangian $L_{f}^{\prime}[Q](t)$ written in the compact form. Finally, the Hamiltonian corresponding to $L_{f}^{\prime}$ is given below

$$
H=\sum_{n=0}^{\infty} P_{(n)}(t) Q^{(n+1)}(t)-L_{f}^{\prime}[Q](t) .
$$

Let us consider the following particular case

$$
L_{f}^{\prime}=\frac{1}{2}\left({ }_{a} \mathbf{D}_{t}^{\alpha} q(t)\right)^{2}+{ }_{a} \mathbf{D}_{t}^{\alpha} F(q(t)) .
$$

By using (9) we obtain

$$
\begin{aligned}
& L_{f}^{\prime} \quad=\quad \frac{1}{2}\left(\frac{(t-a)^{-\alpha}}{\Gamma(1-\alpha)} q(t)+\sum_{k=1}^{\infty}\left(\begin{array}{c}
\alpha \\
k
\end{array}\right) \frac{(t-a)^{k-\alpha}}{\Gamma(k-\alpha+1)} q^{(k)}(t)\right)^{2}+\frac{(t-a)^{-\alpha}}{\Gamma(1-\alpha)} F(q(t)) \\
& +\sum_{k=1}^{\infty}\left(\begin{array}{c}
\alpha \\
k
\end{array}\right) k ! \frac{(t-a)^{k-\alpha}}{\Gamma(k-\alpha+1)} \sum_{s=1}^{k} F^{(s)}(q(t)) \sum \prod_{r=1}^{k} \frac{1}{a_{r} !}\left(\frac{q^{(r)}(t)}{r !}\right)^{a_{r}} .
\end{aligned}
$$

By making in (47) the corresponding substitutions of $\mathrm{q}(\mathrm{t})$ into $Q(x, t)$ we obtain

$$
\begin{aligned}
L_{f}^{\prime}(Q)[t] & =\frac{1}{2}\left(\frac{(t-a)^{-\alpha}}{\Gamma(1-\alpha)} Q(t, x)+\sum_{k=1}^{\infty}\left(\begin{array}{c}
\alpha \\
k
\end{array}\right) \frac{(t-a)^{k-\alpha}}{\Gamma(k-\alpha+1)} Q^{(k)}(t, x)\right)^{2}+\frac{(t-a)^{-\alpha}}{\Gamma(1-\alpha)} F(Q(t, x)) \\
& +\sum_{k=1}^{\infty}\left(\begin{array}{c}
\alpha \\
k
\end{array}\right) k ! \frac{(t-a)^{k-\alpha}}{\Gamma(k-\alpha+1)} \sum_{s=1}^{k} F^{(s)}(Q(t, x)) \sum \prod_{r=1}^{k} \frac{1}{a_{r} !}\left(\frac{Q^{(r)}(t, x)}{r !}\right)^{a_{r}}
\end{aligned}
$$
form

By using (49) the corresponding fractional Euler-Lagrange equation has the

$$
\begin{aligned}
\dot{P}_{(0)} & =\frac{(t-a)^{-\alpha}}{\Gamma(1-\alpha)}\left(\frac{(t-a)^{-\alpha}}{\Gamma(1-\alpha)} Q(t, x)+\sum_{k=1}^{\infty}\left(\begin{array}{c}
\alpha \\
k
\end{array}\right) \frac{(t-a)^{k-\alpha}}{\Gamma(k-\alpha+1)} Q^{(k)}(t, x)\right) \\
& +\frac{(t-a)^{-\alpha}}{\Gamma(1-\alpha)} \frac{d F(Q(t, x))}{d Q(t, x)} \\
& +\sum_{k=1}^{\infty}\left(\begin{array}{c}
\alpha \\
k
\end{array}\right) k ! \frac{(t-a)^{k-\alpha}}{\Gamma(k-\alpha+1)} \sum_{s=1}^{k} \frac{d F^{(s)}(Q(t, x))}{d Q(t, x)} \sum \prod_{r=1}^{k} \frac{1}{a_{r} !}\left(\frac{Q^{(r)}(t, x)}{r !}\right)^{(50)}
\end{aligned}
$$


In the compact form (50) becomes

$$
\dot{P}_{(0)}(t)=\frac{(t-a)^{-\alpha}}{\Gamma(1-\alpha)}{ }_{a} \mathbf{D}_{t}^{\alpha} Q(t, x)+{ }_{a} \mathbf{D}_{t}^{\alpha} \frac{d F(Q(t, x))}{d Q(t, x)} .
$$

The fractional Hamiltonian equations are given below

$$
\begin{aligned}
\dot{P}_{(n)}(t) \quad & +P_{(n-1)}(t)=\left(\frac{(t-a)^{-\alpha}}{\Gamma(1-\alpha)} Q(t, x)+\sum_{k=1}^{\infty}\left(\begin{array}{c}
\alpha \\
k
\end{array}\right) \frac{(t-a)^{k-\alpha}}{\Gamma(k-\alpha+1)} Q^{(k)}(t, x)\right) \\
\times & \left(\begin{array}{c}
\alpha \\
n
\end{array}\right) \frac{(t-a)^{n-\alpha}}{\Gamma(n-\alpha+1)} \\
+\quad & \sum_{k=1}^{\infty}\left(\begin{array}{c}
\alpha \\
k
\end{array}\right) k ! \frac{(t-a)^{k-\alpha}}{\Gamma(k-\alpha+1)} \sum_{s=1}^{k} \frac{d\left(F^{(s)}(Q(t, x)) \sum \prod_{r=1}^{k} \frac{1}{a_{r} !}\left(\frac{Q^{(r)}(t, x)}{r !}\right)^{a_{r}}\right)}{d Q^{(n)}(t, x)}\left(5_{2}\right)
\end{aligned}
$$

and

$$
\dot{Q}^{(n)}(t)=Q^{(n+1)}(t) .
$$

We observe that (52) can be written in the compact form as

$$
\begin{gathered}
\dot{P}_{(n)}(t) \quad+P_{(n-1)}(t)=\left({ }_{a} \mathbf{D}_{t}^{\alpha} Q(t, x)\right)\left(\begin{array}{c}
\alpha \\
n
\end{array}\right) \frac{(t-a)^{n-\alpha}}{\Gamma(n-\alpha+1)} \\
+\quad \frac{d\left({ }_{a} \mathbf{D}_{t}^{\alpha} F(Q(t, x))\right)}{d Q^{(n)}(t, x)}, n \in N .
\end{gathered}
$$

We stress on the fact that $\mathrm{x}$ is not related with space [43]. The negative and the positive values of $\mathrm{x}$ corresponds to interactions with the past and respectively the future [43]. The solution of the chirality condition (53), will give $Q(t, x)=q(x+t)$, which shows the explicit correspondence between $\mathrm{q}(\mathrm{t})$ and the left-mover $Q(x, t)$.

Taking into account that, when $\alpha=1, \Gamma(1)=1$, and using the fact that $\left(\begin{array}{l}1 \\ n\end{array}\right)=0$, for $n>1,{ }_{a} \mathbf{D}_{t}^{\alpha}(Q(t, x))=\frac{d Q(x, t)}{d t}$ and the one-to one correspondence between $q(t)$ and the left mover $Q(x, t)$ we conclude that when the term ${ }_{a} \mathbf{D}_{t}^{\alpha} F(Q(t, x))$ is switched off from (51) and (54), the classical results are reobtained.

\section{Conclusions}

The fractional variational principles are powerful tools used successfully in various area of science and engineering. During the last years several points of view were launched in order to find the fractional Euler-Lagrange equations and to find an appropriate fractional Hamiltonian. One of the key point of these formulations was that under a certain limit the fractional theory includes the classical. As it 
is expected the dynamics of the fractional calculus systems is different from the classical one but the classical dynamics is recovered as a particular case.

In this paper we investigated the Euler-Lagrange and the fractional Hamilton equations corresponding to a fractional generalization of the equivalent Lagrangians. Namely, we add to a given fractional Lagrangian a term which under certain limit reproduces the total derivative at the classical level. We have observed that for the fractional discrete systems the corresponding generalization of the classical equivalent Lagrangians lead us to a theory possessing infinite higher order derivatives. We have calculated the fractional Euler-Lagrangian for a particular case as well as the fractional Hamilton equations were obtained in a most general case.

We consider two specific examples in this paper. For the first example we consider one of the possible fractional generalization of the free one dimensional particle Lagrangian and we add to it a term of the form $C_{a} \mathbf{D}_{t}^{\alpha} q(t)$. From the classical point of view the above investigated Lagrangians are equivalent. Both fractional Euler-Lagrange and Hamilton equations are obtained for this example and it was proved that they are equivalent in the fractional case. For example, the difference between the classical case and the fractional one is illustrated by the form of the fractional Euler-Lagrange equations given by (27). The second example deals with the same fractional generalization of the free one dimensional particle but we add a term of ${ }_{a} \mathbf{D}_{t}^{\alpha} F(q(t))$. In this case, due to the Faà di Bruno formula, we used the $1+1$ field formalism in order to obtain the corresponding fractional Euler-Lagrange and Hamilton equations. The classical results are reobtained when $\alpha=1$.

\section{Acknowledgments}

One of the authors (D.B.) would like to thank J. Cresson, F. Mainardi for reading the manuscript. The authors would like to thank J. J. Trujillo for continuing support and for interesting discussions. This work is partially supported by the Scientific and Technical Research Council of Turkey.

\section{References}

[1] Samko, S. G., Kilbas, A. A., Marichev, O. I.: Fractional Integrals and Derivatives - Theory and Applications. Gordon and Breach, Linghorne, P.A.(1993).

[2] Podlubny, I.: Fractional Differential Equations. Academic Press, New York (1999).

[3] Kilbas, A. A., Srivastava, H. M., Trujillo, J. J.: Theory and Applications of Fractional Differential Equations. Elsevier (2006). 
[4] Tenreiro Machado, J. A.: A probabilistic interpretation of the fractional-order differentiation. Frac. Calc. Appl. Anal. 8, 73-80 (2003).

[5] Tenreiro Machado, J. A.: Discrete-time fractional-order controllers. Frac. Calc. Appl. Anal. 4, 47-66 (2001).

[6] Lorenzo, C. F., Hartley, T. T.: Fractional trigonometry and the spiral functions. Nonlinear Dynamics.38, 23-60 (2004).

[7] Agrawal, O. P.: Application of fractional derivatives in thermal analysis of disk brakes. Nonlinear Dynamics. 38, 191-206 (2004).

[8] Silva, M. F., Tenreiro Machado, J. A., Lopes, A. M.: Modelling and simulation of artificial locomotion systems.Robotica. 23, 595-606 (2005).

[9] Agrawal, O. P.: A general formulation and solution scheme for fractional optimal control problems. Nonlinear Dynamics. 38, 323-337 (2004).

[10] Gorenflo, R., Mainardi, F.: Fractional Calculus: Integral and Differential Equations of Fractional Orders, Fractals and Fractional Calculus in Continoum Mechanics. Springer Verlag, Wien and New York (1997).

[11] Mainardi, F.: Fractional relaxation-oscillation and fractional diffusion-wave phenomena. Chaos, Solitons and Fractals.7(9), 1461-1477 (1996).

[12] Mainardi, F., Pagnini, G., Gorenflo, R.: Mellin transform and subordination laws in fractional diffusion processes. Frac. Calc. Appl. Anal. 6(4), 441-459 (2003).

[13] Raspini, A.: Simple solutions of the fractional Dirac equation of order $\frac{2}{3}$. Physica Scripta. 64, 20-22 (2001).

[14] Rabei,E., Alhalholy, T., Rousan, A.: Potential of arbitrary forces with fractional derivatives. Int. J. Theor. Phys. A. 19(17-18) 3083-3096 (2004)

[15] Naber, M.: Time fractional Schrödinger equation. J. Math. Phys., 45(8), 3339-3352 (2004).

[16] Lim , S. C., Muniady, S. V.: Stochastic quantization of nonlocal fields. Physics Letters A. 324, 396-405 (2004).

[17] Magin, R. L.: Fractional calculus in bioengineering. Critic. Rev. in Biomed. Eng.32(1), 1-104 (2004).

[18] Magin, R. L.: Fractional Calculus in Bioengineering, Part 2. Critic. Rev. in Biomed. Eng. 32(2), 105-193 (2004). 
[19] Magin, R. L.: Fractional Calculus in Bioengineering, Part 3. Critic. Rev. in Biomed. Eng. 32(3/4), 194-377 (2004).

[20] Riewe, F.: Nonconservative Lagrangian and Hamiltonian mechanics, Phys. Rev. E. 53, 1890-1899 (1996).

[21] Riewe, F.: Mechanics with fractional derivatives, Phys. Rev. E 55, 3581-3592 (1997).

[22] Agrawal, O. P.: Formulation of Euler-Lagrange equations for fractional variational problems. J. Math. Anal. Appl. 272, 368-379 (2002).

[23] Klimek, M.: Fractional sequential mechanics-models with symmetric fractional derivatives. Czech. J. Phys. 51: 1348-1354 (2001). Klimek, M.: Lagrangian and Hamiltonian fractional sequential mechanics. Czech. J. Phys. $52,1247-1253$ (2002).

[24] Cresson, J.: Fractional embedding of differential operators and Lagrangian systems, Preprint, (2006).

[25] Baleanu, D., Muslih, S.: Lagrangian formulation of classical fields within Riemann-Liouville fractional derivatives. Physica Scripta. 72(2-3), 119-121 (2005).

[26] Muslih, S., Baleanu, D.: Hamiltonian formulation of systems with linear velocities within Riemann-Liouville fractional derivatives. J. Math. Anal. Appl. 304(3), 599-606 (2005).

[27] Rabei, E. M., Nawafleh, K.I., Hijjawi, R. S., Muslih, S. I., Baleanu, D.: The Hamilton formalism with fractional derivatives, in press, J. Math. Anal. Appl., 2007.

[28] Baleanu, D., Muslih, S.I.: Formulation of Hamiltonian equations for fractional variational problems. Czech. J. Phys. 55 (6), 633-642 (2005).

[29] Muslih, S., Baleanu, D., Rabei, E.: Hamiltonian formulation of classical fields within Riemann-Liouville. Physica Scripta 73(6),436-438, (2006).

[30] Baleanu, D., Avkar, T.: Lagrangians with linear velocities within RiemannLiouville fractional derivatives. Nuovo Cimento B. 119, 73-79 (2004).

[31] Baleanu, D.: Constrained systems and Riemann-Liouville fractional derivative. In Proceedings of $1^{\text {st }}$ IFAC Workshop on Fractional Differentiation and its Applications,pp. 597-602, Bordeaux, France, July 19-21 (2004).

[32] Baleanu, D.: About fractional calculus of singular Lagrangians, in Proceedings of IEEE International Conference on Computational Cybernetics ICCC, pages 379-385, Wien, Austria, August 30-September 1 (2004). 
[33] Baleanu, D., Muslih, S.: New trends in fractional quantization method.Intelligent Systems at the Service of Mankind, volume 2. U-Books Verlag, Augsburg, Germany, 1st Edition, December 2005.

[34] Baleanu, D.: Constrained systems and Riemann-Liouville fractional derivatives. Fractional differentiation and its applications. U-Books Verlag, Augsburg, Germany, (A. Le Mehaute, J.A. Tenreiro Machado, J.C. Trigeassou, J. Sabatier, Eds.),pp. 69-80, 2005.

[35] Baleanu, D., Muslih,S. I.: About fractional supersymmetric quantum mechanics. Czech. J.Phys. 55(9), 1063-1066 (2005).

[36] Henneaux, M., Teitelboim, C.: Quantization o Gauge Systems. Princeton University.(1993).

[37] Abramowitz, M., Stegun,I. A.: Handbook of Mathematical Functions, Nauka, Moscow (1979).

[38] Hardy, G. H.: Riemann's form of Taylor's series. J. London Math. Soc. 20, 48-57 (1954).

[39] Trujillo, J. J.: On a Riemann-Liouville generalized Taylors formula. J. Math. Anal. Appl. 231, 255265 (1999).

[40] Llosa, J., Vives, J.: Hamiltonian formalism for nonlocal Lagrangians. J. Math. Phys. 35, 2856-2877 (1994).

[41] Gitman, D. M., Tytin, I. V.: Quantization of fields with constraints. Springer-Verlag (1990).

[42] Nesterenko, V. V.: Singular Lagrangians with higher derivatives. J. Phy A: Math. Gen.22, 1673-1687 (1989).

[43] Bering, K.: A note of non-locality and Ostrogradski's construction, preprint hep-th/0007192.

[44] Gomis, J., Kamimura, K., Llosa, J.:Hamiltonian formalism for space-time noncummutative theories.Phys. Rev. D 63,045003-1-945003-6 (2001).

[45] Carathéodory,C.: Calculus of Variations and Partial Differential Equations of First Order, Part II, Holden-Day (1967).

[46] Negri, L. J., da Silva Joseph, E. G. : s-equivalent Lagrangians in generalized mechanics. Phys. Rev. D 27, 2227-2232 (1986).

[47] Santilli, M.: Foundations of Theoretical Mechanics I, II, Springer, New York (1977). 